\title{
Proposition d'extension du modèle génétique additif classique à des situations non panmictiques
}

\author{
B. LANGLOIS \\ I.N.R.A., Station de Génétique quantitative et appliquée, \\ Centre de Recherches zootechniques \\ F 78350 Jouy-en-Josas (France)
}

\begin{abstract}
Résumé
Les résultats de FISHER (1918) sur la corrélation entre apparentés ont été étendus à un certain nombre de situations souvent non prises en compte comme les croisements raisonnés dans une population sélectionnée et une répartition non au hasard des effets du milieu.

Dans un premier temps en ne tenant compte que de la variabilité génétique additive, les formules d'estimation de l'héritabilité déduites du travail de FisHer sont confirmées et étendues à une plus large gamme de situations.

Dans un second temps, l'introduction d'une corrélation génotype-milieu permet d'apprécier son incidence sur les estimations précédentes.

On conclut sur l'utilité de la formulation d'un tel modèle qui amène à clarifier les hypothèses génétiques et conduit ainsi à une meilleure approche des problèmes d'indexation. Dans les cas où l'on ne peut délibérément écarter l'existence d'une corrélation génotypemilieu, il permet parfois de l'estimer et de toute façon permet d'apprécier son incidence éventuelle sur les résultats. Cette approche conduit d'ailleurs à examiner de façon originale l'analyse de certains effets maternels.
\end{abstract}

\section{Introduction}

La discipline de la "génétique quantitative» est de plus en plus souvent sujette à toutes sortes de critiques : génétique trop simple ou statistique trop compliquée, il est en effet facile a priori d'en dénoncer les défauts. Elle n'en demeure pas moins le seul ensemble de techniques cohérentes pour pratiquer l'amélioration génétique des espèces végétales et animales de l'agriculture. Elle le doit sans doute à la remarquable simplicité du modèle génétique classique et à la sophistication de plus en plus grande de l'outil mathématique utilisé pour le traiter.

Pour illustrer ce thème, nous nous proposons d'étendre l'application du modèle génétique additif classique à un certain nombre de situations souvent non prises en compte comme les croisements raisonnés dans une population sélectionnée et une répartition non au hasard des effets du milieu. C'est l'analyse génétique des performances réalisées par les chevaux de Pur Sang (LangloIs, 1975, 1980) qui nous a 
conduits à approfondir cette question qui se rapproche des problèmes soulevés par l'analyse des résultats des tests de QI chez l'Homme (JENCKs, 1972) et qui peut également, nous le verrons, trouver d'autres applications en zootechnie.

\section{Position du problème}

Le phénotype est le résultat de l'action conjointe des gènes et du milieu. Cette donnée d'expérience peut se traduire par des modèles mathématiques plus ou moins sophistiqués qui sont une façon schématique de traduire la réalité. On écrit généralement :

$$
\mathbf{P}=\mathbf{A}+\mathbf{R} \text { avec } \operatorname{Cov}(\mathbf{A}, \mathbf{R})=\mathbf{0}
$$

$\mathbf{P}$ est la valeur phénotypique d'un individu (sa mesure).

A est sa valeur génétique additive.

$\mathbf{R}$ est la résiduelle due essentiellement aux effets du milieu.

Cela suppose l'hérédité additive du caractère et l'absence de corrélation ou d'interaction génotype-milieu. C'est le modèle qui sert le plus souvent en zootechnie et qui est le fondement de toutes les estimations de valeurs génétiques. C'est lui qui confère au paramètre héritabilité $h^{2}=\operatorname{Var}(A) / \operatorname{Var}(P)$ toute sa valeur prédictive. En effet, puisque $\operatorname{Cov}(A, R)=0, \operatorname{Cov}(A, P)=\operatorname{Var}(A)$. Il s'ensuit que la corrélation entre $\mathrm{A}$ et $\mathrm{P}$ est égale à $\mathrm{h}$, le coefficient de régression linéaire de $\mathrm{A}$ en $\mathrm{P}$ est égal à $\mathrm{h}^{2}$ et celui de $\mathrm{P}$ en $\mathrm{A}$ est égal à 1.

Symétriquement $\operatorname{Cov}(\mathrm{R}, \mathrm{P})=\operatorname{Var}(\mathrm{R})$ et puisque $\operatorname{Var}(\mathrm{P})=\operatorname{Var}(\mathrm{A})+\operatorname{Var}(\mathrm{R})$ le coefficient de régression linéaire de $R$ en $P$ est égal à $1-h^{2}$.

Or, nous savons qu'un individu reçoit la moitié de ses gènes de chacun de ses deux parents. Nous poserons donc que l'espérance de la valeur génétique additive d'un individu est égale à la moitié de celle de son père plus la moitié de celle de sa mère.

$$
\operatorname{Esp}\left(A_{d} / A_{p}, A_{m}\right)=\frac{1}{2} A_{p}+\frac{1}{2} A_{m}
$$

Cela conduit sans faire les autres hypothèses habituelles aux relations suivantes entre les variances et covariances des valeurs génétiques :

- Relation père-descendant :

$$
\operatorname{Cov}\left(A_{p}, A_{d}\right)=\frac{1}{2} \operatorname{Var}\left(A_{p}\right)+\frac{1}{2} \operatorname{Cov}\left(A_{p}, A_{m}\right)
$$

Les indices $\mathrm{p}, \mathrm{m}$ et $\mathrm{d}$ affectent les valeurs génétiques $\mathrm{A}$ respectivement au père, à la mère et au descendant.

- Relation mère-descendant :

$$
\operatorname{Cov}\left(A_{m}, A_{d}\right)=\frac{1}{2} \operatorname{Var}\left(A_{m}\right)+\frac{1}{2} \operatorname{Cov}\left(A_{p}, A_{m}\right)
$$


- Relation entre descendants du même père :

$$
\operatorname{Cov}\left(A_{d}, A_{d^{\prime}}\right)=\frac{1}{4} \operatorname{Var}\left(A_{p}\right)+\frac{1}{2} \operatorname{Cov}\left(A_{p}, A_{m}\right)+\frac{1}{4} \operatorname{Cov}\left(A_{m}, A_{m^{\prime}}\right)
$$

Les deux descendants considérés sont distingués par un signe prime (') comme leurs mères respectives.

- Relation entre frères utérins :

$$
\operatorname{Cov}\left(A_{d}, A_{d^{\prime}}\right)=\frac{1}{4} \operatorname{Cov}\left(A_{p}, A_{p^{\prime}}\right)+\frac{1}{2} \operatorname{Cov}\left(A_{p}, A_{m}\right)+\frac{1}{4} \operatorname{Var}\left(A_{m}\right)
$$

Les deux pères considérés sont distingués par un signe prime (').

Nous voyons ainsi apparaître des termes qui en panmixie sont nuls par définition comme $\operatorname{Cov}\left(A_{p}, A_{m}\right)$, $\operatorname{Cov}\left(A_{m}, A_{m}\right)$ et $\operatorname{Cov}\left(A_{p}, A_{p^{\prime}}\right)$ ou d'autres qui n'ont pas de raison d'être distingués en l'absence de sélection mais qui peuvent différer sensiblement en sa présence tels $\operatorname{Var}\left(A_{p}\right)$ et $\operatorname{Var}\left(A_{m}\right)$.

Le rôle du généticien est de formuler dans chaque cas les hypothèses convenables qui permettent d'estimer ces variances de valeurs génétiques en fonction des variances et covariances phénotypiques correspondantes qui sont les seules mesurables.

\section{Analyse des relations entre parents et descendants : estimation de l'héritabilité}

Si l'on suppose que la covariance phénotypique entre conjoints est uniquement due à l'assortiment des phénotypes, ce qui, en termes d'espérance conditionnelle, s'écrit :

$$
\begin{array}{ll} 
& \operatorname{Esp}\left(A_{p} / P, M\right)=\operatorname{Esp}\left(A_{p} / P\right) \\
& \operatorname{Esp}\left(A_{m} / P, M\right)=\operatorname{Esp}\left(A_{m} / M\right) \\
\text { et } & \operatorname{Esp}\left(A_{p} A_{m} / P, M\right)=\operatorname{Esp}\left(A_{p} / P\right) \times \operatorname{Esp}\left(A_{m} / M\right)
\end{array}
$$

L'expression :

$$
\operatorname{Cov}(P, M)=\operatorname{Cov}\left(A_{p}, A_{m}\right)+\operatorname{Cov}\left(A_{p}, R_{m}\right)+\operatorname{Cov}\left(A_{m}, R_{p}\right)+\operatorname{Cov}\left(R_{p}, R_{m}\right)
$$

pourrra se décomposer en :

$$
\begin{aligned}
& \operatorname{Cov}\left(A_{p}, A_{m}\right)=\underset{h_{p}^{2}}{2}{ }^{2} \operatorname{Cov}(P, M) \\
& \operatorname{Cov}\left(A_{p}, R_{m}\right)=\underset{h_{p}}{2}\left(1-h_{m}^{2}\right) \operatorname{Cov}(P, M) \\
& \operatorname{Cov}\left(A_{m}, R_{p}\right)=\stackrel{h_{m}}{2}\left(1-\underset{h_{p}}{2} \operatorname{Cov}(P, M)\right. \\
& \operatorname{Cov}\left(R_{p}, R_{m}\right)=\left(1-h_{p}^{2}\right)\left(1-h_{m}^{2}\right) \operatorname{Cov}(P, M)
\end{aligned}
$$

On pourra exprimer dans le détail les covariances entre parents et descendants qui découlent du modèle génétique proposé.

Dans la notation, $P, M$ et $D$ sont les valeurs phénotypiques d'un père, d'une mère et d'un descendant.

$$
\mathbf{h}_{\mathrm{p}}^{2}=\operatorname{Var}\left(\mathrm{A}_{\mathrm{p}}\right) / \operatorname{Var}(\mathrm{P}) \text { et } \mathrm{h}_{\mathrm{m}}^{2}=\operatorname{Var}\left(\mathrm{A}_{\mathrm{m}}\right) / \operatorname{Var}(\mathrm{M})
$$


De plus pour simplifier l'écriture, on posera par la suite :

$$
\operatorname{Var}(P)=V_{p}, \operatorname{Var}(M)=V_{m} \text { et } \operatorname{Var}(D)=V_{d}
$$

Pour estimer l'héritabilité, les relations de la figure 1 montrent qu'on ne peut formuler que trois types d'hypothèses car $\operatorname{Cov}\left(A_{d}, R_{n}\right) \operatorname{comme} \operatorname{Cov}\left(A_{d}, R_{m}\right)$ ne peuvent être supposées nulles à cause de l'homogamie phénotypique.

On voit en effet que par exemple $R_{p}$ et $A_{d}$ sont corrélées par les chemins $R_{p} \leftrightarrow A_{m} \rightarrow A_{d}$ et $R_{p} \leftrightarrow R_{m} \leftrightarrow A_{p} \rightarrow A_{d}$.

Hypothèse 1 : la liaison entre l'effet du milieu sur le descendant et la valeur génétique des parents est nulle, soit :

$$
\operatorname{Cov}\left(A_{p}, R_{d}\right)=\operatorname{Cov}\left(A_{m}, R_{d}\right)=0
$$

Hypothèse 2 : la liaison entre les effets du milieu sur les parents et sur les descendants est nulle, soit :

$$
\operatorname{Cov}\left(\mathbf{R}_{\mathrm{p}}, \mathbf{R}_{\mathrm{d}}\right)=\operatorname{Cov}\left(\mathbf{R}_{\mathrm{m}}, \mathbf{R}_{\mathrm{d}}\right)=0
$$

Hypothèse 3 : la liaison entre l'effet du milieu sur le descendant et le phénotype des parents est nulle, soit :

$$
\operatorname{Cov}\left(\mathbf{R}_{\mathrm{d}}, \mathbf{P}\right)=\operatorname{Cov}\left(\mathbf{R}_{\mathrm{d}}, \mathrm{M}\right)=0
$$

Or on peut vérifier (figure 1) que si l'une quelconque de ces covariances n'est pas nulle, il n'y a pas de raison de supposer que les autres le soient. Les corrélations introduites par l'hypothèse de décomposition de $\operatorname{Cov}(\mathrm{P}, \mathrm{M})$ (homogamie phénotypique) les rendent en effet toutes solidaires. Les covariances impliquées dans les hypothèses 1 , 2 et 3 sont donc soit toutes nulles soit toutes non nulles et dans ce dernier cas le problème de la corrélation génotype-milieu se pose.

L'hypothèse 3 supposant 1 et 2 réalisées est donc la seule qui soit cohérente avec le modèle d'analyse décrit par la figure 1. On peut toutefois avec d'autres hypothèses de décomposition de $\operatorname{Cov}(\mathrm{P}, \mathrm{M})$ correspondant à des situations plus particulières formuler d'autres hypothèses, mais là n'est pas notre propos.

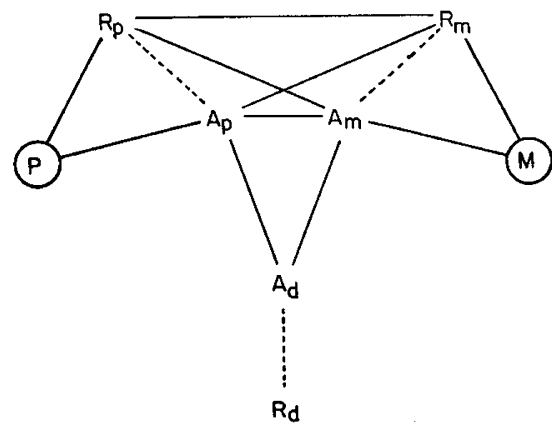

FIG. 1

Réseau des corrélations postulées dans l'analyse des relations entre parents et descendants en l'absence de corrélation génotype-milieu

Network of the assumed correlations in the analysis of the parent-offspring relationships without genotype-environment correlation

Corrélations postulées non nulles par le modèle d'analyse proposé.

Correlations assumed to be non null by the proposed model of analysis

Corrélations postulées nulles.

Correlations assumed to be null. 
Nous supposerons que :

$$
\begin{array}{ll} 
& \operatorname{Cov}\left(\mathrm{A}_{\mathrm{d}}, \mathrm{P}\right)=\operatorname{Cov}(\mathrm{P}, \mathrm{D}) \\
\text { et } \quad & \operatorname{Cov}\left(\mathrm{A}_{\mathrm{d}}, \mathrm{M}\right)=\operatorname{Cov}(\mathrm{M}, \mathrm{D})
\end{array}
$$

qui, sous l'hypothèse génétique $\operatorname{Esp}\left(A_{d} / A_{p}, A_{m}\right)=\frac{1}{2} A_{p}+\frac{1}{2} A_{m}$ conduisent à :

$$
\begin{aligned}
\operatorname{Cov}(P, D) & =\frac{1}{2} \operatorname{Cov}\left(A_{p}, P\right)+\frac{1}{2} \operatorname{Cov}\left(A_{m}, P\right) \\
\text { et } \quad \operatorname{Cov}(M, D) & =\frac{1}{2} \operatorname{Cov}\left(A_{m}, M\right)+\frac{1}{2} \operatorname{Cov}\left(A_{p}, M\right)
\end{aligned}
$$

qui, sachant que sous l'hypothèse d'additivité $\operatorname{Esp}\left(A_{\mathfrak{p}} / P\right)=\stackrel{h_{p} P}{2}$ et $\operatorname{Esp}\left(A_{m} / M\right)=h_{m}^{2} M$ donne le système d'estimation de $\stackrel{2}{h_{p}}$ et ${ }_{h_{m}}^{2}$ suivant :

$$
\left\{\begin{array}{l}
\operatorname{Cov}(\mathrm{P}, \mathrm{D})=\frac{1}{2} \mathrm{~h}_{\mathrm{p}}^{2} \mathrm{~V}_{\mathrm{p}}+\frac{1}{2} \mathrm{~h}_{\mathrm{m}}^{2} \operatorname{Cov}(\mathrm{P}, \mathrm{M}) \\
\operatorname{Cov}(\mathrm{M}, \mathrm{D})=\frac{1}{2} \mathrm{~h}_{\mathrm{m}}^{2} \mathrm{~V}_{\mathrm{m}}+\frac{1}{2} \mathrm{~h}_{\mathrm{p}}^{2} \operatorname{Cov}(\mathrm{P}, \mathrm{M})
\end{array}\right.
$$

On peut en effet noter que à $A_{p}$ et $A_{m}$ constants $P$ et $D$ (respectivement $M$ et $D$ ) deviennent indépendants du fait de nos hypothèses.

Les solutions $\mathrm{h}_{\mathrm{p}}^{2}$ et $\mathrm{h}_{\mathrm{m}}^{2}$ sont les doubles des coefficients de régression partiels du phénotype du descendant en celui du père à mère constante $\left(b_{d / p . m}\right)$ et du phénotype du descendant en celui de la mère à père constant $\left(b_{d / m . p}\right)$ soit :

$$
\begin{aligned}
\stackrel{2}{h_{p}} & =2 b_{d / p . m} \\
h_{m}^{2} & =2 b_{d / m . p}
\end{aligned}
$$

Par ailleurs si l'on suppose $\mathrm{h}_{\mathrm{p}}^{2}=\mathrm{h}_{\mathrm{m}}^{2}=\mathrm{h}^{2}$ ce qui n'est pas une hypothèse très lourde, les deux équations donnent l'identité suivante :

$$
h^{2}=\frac{2 \operatorname{Cov}(P, D)}{V_{p}+\operatorname{Cov}(P, M)}=\frac{2 \operatorname{Cov}(M, D)}{V_{m}+\operatorname{Cov}(P, M)}
$$

qui doit être vérifiée si $\operatorname{Cov}\left(P, R_{d}\right)=\operatorname{Cov}\left(M, R_{d}\right)$ et en particulier si elles sont toutes les deux nulles. Ces deux équations si $\mathrm{V}_{\mathrm{p}}=\mathrm{V}_{\mathrm{m}}$ correspondent à la relation donnée par FisHER (1918) pour la corrélation parent-descendant.

Par ailleurs elles permettent de retrouver le résultat classique (REEve, 1953-1955) de la régression du descendant en son parent moyen $\overline{\mathrm{P}}$ soit :

$$
\mathrm{h}^{2}=\operatorname{Cov}(\mathrm{D}, \overline{\mathrm{P}}) / \operatorname{Var}(\overline{\mathrm{P}})
$$


Cela montre que les hypothèses sous-jacentes à cette estimation sont également une indépendance des effets du milieu sur le descendant vis-à-vis des phénotypes parentaux.

\section{Analyse des relations entre demi et pleins frères : estimation de l'héritabilité}

Définissons tout d'abord quelques notations. Soient :

$t_{m}$ : la corrélation intra classe de père du phénotype des mères ;

$u_{p}$ : la corrélation intra classe de mère du phénotype des pères ;

$t_{d}$ : la corrélation intra classe de père du phénotype des descendants ;

$v_{\mathrm{d}}$ : la corrélation intra classe de couple père-mère du phénotype des descendants ;

$\mathrm{u}_{\mathrm{d}}$ : la corrélation intra classe de mère du phénotype des descendants.

Nous pourrons écrire dans le cas des descendants d'un même père :

$$
\begin{array}{ll} 
& \operatorname{Cov}\left(D, D^{\prime}\right)=t_{d} V_{a d} \\
\text { et } & \operatorname{Cov}\left(M, M^{\prime}\right)=t_{m} V_{m}
\end{array}
$$

dans celui de plein-frères :

$$
\operatorname{Cov}\left(\mathrm{D}, \mathrm{D}^{\prime}\right)=\mathrm{v}_{\mathrm{d}} \mathrm{V}_{\mathrm{a}}
$$

et dans celui de descendants d'une même mère :

$$
\begin{array}{ll} 
& \operatorname{Cov}\left(D, D^{\prime}\right)=u_{d} V_{d} \\
\text { et } \quad & \operatorname{Cov}\left(P, P^{\prime}\right)=u_{p} V_{p}
\end{array}
$$

Si l'on veut supposer comme pour $\operatorname{Cov}(P, M)$ que $\operatorname{Cov}\left(M, M^{\prime}\right)$ n'est dû qu'à l'assortiment du phénotype des mères, on pourra estimer $\operatorname{Cov}\left(A_{m}, A_{m}\right.$ ') par $h_{m}^{4} t_{m} V_{m}$ et $\operatorname{Cov}\left(A_{p}, A_{p^{\prime}}\right)$ par $h_{p}^{4} u_{p} V_{p}$. Ces hypothèses mènent aux expressions des composantes de la variance utilisées pour estimer $h^{2}$. On peut toutefois noter que $\operatorname{Cov}\left(A_{m}, A_{m^{\prime}}\right)$ et surtout $\operatorname{Cov}\left(A_{p}, A_{p^{\prime}}\right)$ risquent souvent d'être sous-estimées. La première dans le cas fréquent d'apparentement des mères, l'assortiment est alors plus génétique que phénotypique; la seconde du fait que les pères auxquels une mère est accouplée sont le plus souvent d'une valeur génétique très comparable. On peut dans ces cas-là en posant $\operatorname{Cov}\left(A_{m}, A_{m^{\prime}}\right)=t_{m} V_{m}$ ou $\operatorname{Cov}\left(A_{p}, A_{p^{\prime}}\right)=u_{p} V_{p}$ maximiser leurs estimations ce qui permet d'encadrer les valeurs recherchées.

L'estimation de l'héritabilité par les méthodes d'analyse de la variance est réalisée lorsque l'on peut supposer que l'essentiel de la ressemblance entre frère ou demi-frères n'est due qu'à leur héritage génétique. On fait alors l'hypothèse :

qui conduit à :

$$
\operatorname{Cov}\left(A_{d}, R_{d^{\prime}}\right)=\operatorname{Cov}\left(R_{d^{l}}, R_{d^{\prime}}\right)=0
$$

$$
\operatorname{Cov}\left(D, D^{\prime}\right)=\operatorname{Cov}\left(A_{d}, A_{d^{\prime}}\right)
$$


Dans le cas de descendants du même père nous obtenons :

soit :

$$
\operatorname{Cov}\left(D, D^{\prime}\right)=\frac{1}{4} \operatorname{Var}\left(A_{p}\right)+\frac{1}{2} \operatorname{Cov}\left(A_{p}, A_{m}\right)+\frac{1}{4} \operatorname{Cov}\left(A_{m}, A_{m^{\prime}}\right)
$$

$$
t_{d} V_{d}=\frac{1}{4} h_{p}^{2} V_{p}+\frac{1}{2} h_{p}^{2} h_{m}^{2} \operatorname{Cov}(P, M)+\frac{1}{4} h_{m}^{4} t_{m} V_{m}
$$

qui, si nous supposons $h_{p}^{2}=h_{m}^{2}=h^{2}$ conduit à une équation du second degré en $h^{2}$.

$$
h^{4}\left[2 \operatorname{Cov}(P, M)+t_{m} V_{m}\right]+h^{2} V_{p}-4 V_{d} t_{d}=0
$$

ou encore :

$$
h^{4}\left[2 b_{p / m}+t_{m}\right]+h^{2} \frac{V_{p}}{V_{n}}-4 \frac{V_{d}}{V_{m}} t_{d}=0
$$

dont la racine positive fournira l'estimation recherchée.

De la même façon si l'on peut supposer l'absence d'effets maternels, nous obtiendrons dans le cas de frères utérins :

$$
h^{4}\left[2 b_{p / m}+\frac{V_{v}}{V_{m}} u_{p}\right]+h^{2}-4 \frac{V_{d}}{V_{m}} u_{d}=0
$$

Dans le cas de plein frères sous les mêmes hypothèses :

$$
\operatorname{Cov}\left(D . D^{\prime}\right)=\frac{1}{4} \operatorname{Var}\left(A_{p}\right)+\frac{1}{2} \operatorname{Cov}\left(A_{p}, A_{m}\right)+\frac{1}{4} \operatorname{Var}\left(A_{m}\right)
$$

d'où :

soit :

$$
4 v_{d} V_{d}=h^{2} V_{p}+2 h^{4} \operatorname{Cov}(P, M)+h^{2} V_{m}
$$

$$
h^{4} 2 b_{p / m}+h^{2}\left(1+\frac{V_{v}}{V_{m}}\right)-4 v_{d} \frac{V_{d}}{V_{m}}=0
$$

qui si l'on fait abstraction de la dominance correspond également à l'expression de la corrélation entre frères donnée par FISHER (1918).

On peut en outre vérifier qu'en panmixie $\operatorname{Cov}(P, M)=0$ et en l'absence de sélection $V_{p}=V_{m}=V_{d}$ on retrouve bien selon les cas $h^{2}=4 t_{d}, h^{2}=4 u_{d}$ et $\mathbf{h}^{2}=2 \mathrm{v}_{\mathrm{d}}$.

\section{Introduction d'une corrélation génotype-milieu}

Si l'on introduit une corrélation s entre la valeur génétique $\mathrm{A}$ et la résidiuelle $\mathrm{R}$,

$$
\operatorname{Var}(\mathbf{P})=\operatorname{Var}(\mathrm{A})+2 \operatorname{Cov}(\mathbf{A}, \mathbf{R})+\operatorname{Var}(\mathbf{R})
$$




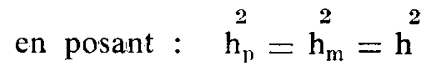

$$
\begin{aligned}
& \text { avec } \mathrm{h}^{2}=\operatorname{Var}(\mathrm{A}) / \operatorname{Var}(\mathrm{P}) \text { et } \mathrm{e}^{2}=\operatorname{Var}(\mathrm{R}) / \operatorname{Var}(\mathrm{P})
\end{aligned}
$$

cela se traduit par :

$$
\mathrm{h}^{2}+2 \text { hes }+\mathrm{e}^{2}=1
$$

On s'aperçoit que l'héritabilité perd la valeur prédictive qu'elle possédait car :

$$
\operatorname{Cov}(\mathrm{A}, \mathrm{P}) / \operatorname{Var}(\mathrm{P})=\mathrm{h}(\mathrm{h}+\mathrm{es})
$$

et :

$$
\operatorname{Cov}(\mathrm{R}, \mathrm{P}) / \operatorname{Var}(\mathrm{P})=\mathrm{e}(\mathrm{e}+\mathrm{hs})
$$

Si comme précédemment on suppose au niveau de la structure de reproduction un assortiment sur lies phénotypes exclusivement on aura les équations suivantes :

$$
\begin{aligned}
& \operatorname{Cov}\left(A_{p}, A_{m}\right)=h^{2}(h+e s)^{2} \operatorname{Cov}(P, M) \\
& \operatorname{Cov}\left(A_{p}, R_{m}\right)=h e(h+e s)(e+h s) \operatorname{Cov}(P, M)=\operatorname{Cov}\left(A_{m}, R_{p}\right) \\
& \operatorname{Cov}\left(R_{p}, R_{m}\right)=e^{2}(e+h s)^{2} \operatorname{Cov}(P, M) \\
& \operatorname{Cov}\left(A_{m}, A_{m^{\prime}}\right)=h^{2}(h+e s)^{2} t_{m} V_{m} \\
& \operatorname{Cov}\left(A_{m}, R_{m^{\prime}}\right)=h e(h+e s)(e+h s) t_{m} V_{m}=\operatorname{Cov}\left(A_{m^{\prime}}, R_{m}\right) \\
& \operatorname{Cov}\left(R_{m}, R_{m^{\prime}}\right)=e^{2}(e+h s)^{2} t_{m} V_{m} \\
& \operatorname{Cov}\left(A_{p}, A_{p^{\prime}}\right)=h^{2}(h+e s)^{2} u_{p} V_{p} \\
& \operatorname{Cov}\left(A_{p}, A_{p^{\prime}}\right)=h e(h+e s)(e+h s) u_{p} V_{p}=\operatorname{Cov}\left(A_{p^{\prime}}, R_{p}\right) \\
& \operatorname{Cov}\left(R_{p}, R_{p^{\prime}}\right)=e^{2}(e+h s)^{2} u_{p} V_{p}
\end{aligned}
$$

Que deviennent les estimations de l'héritabilité dans ce nouveau modèle décrit par la figure 2 .

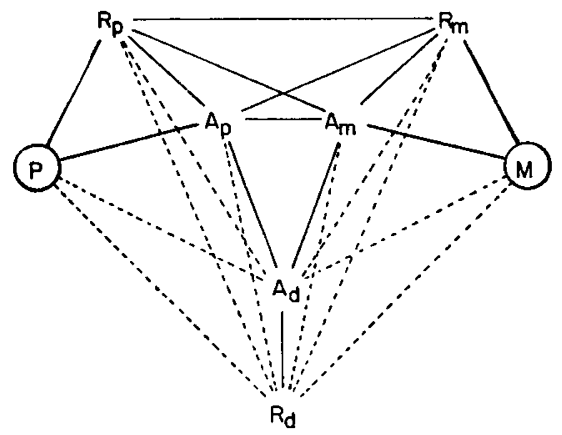

FIG. 2

Réseau de l'ensemble des corrélations impliquées dans l'analyse des relations entre parents et descendants en présence d'une corrélation génotype-milieu

Network of all the correlations involved in the analysis of the parent-offspring relationships with a genotype-environment correlation

Corrélations postulées par le modèle d'analyse.

Correlations assumed by the model of analysis.

Corrélations dérivant des précédentes.

Correlations resulting from the former. 


$$
\begin{gathered}
\operatorname{Cov}(\mathbf{P}, \mathrm{D})=\operatorname{Cov}\left(\mathbf{A}_{d}, \mathbf{P}\right)+\operatorname{Cov}\left(\mathbf{R}_{\mathrm{d}}, \mathbf{P}\right) \\
\operatorname{Cov}(\mathbf{M}, \mathrm{D})=\operatorname{Cov}\left(\mathbf{A}_{\mathrm{d}}, \mathbf{M}\right)+\operatorname{Cov}\left(\mathbf{R}_{\mathrm{d}}, \mathbf{M}\right)
\end{gathered}
$$

sous l'hypothèse génétique $\operatorname{Esp}\left(A_{d} / A_{p}, A_{m}\right)=\frac{1}{2} A_{p}+\frac{1}{2} A_{m}$, on a :

$$
\begin{aligned}
& \operatorname{Cov}\left(A_{d}, P\right)=\frac{1}{2} \operatorname{Cov}\left(A_{p}, P\right)+\frac{1}{2} \operatorname{Cov}\left(A_{m}, P\right) \\
& \operatorname{Cov}\left(A_{d}, M\right)=\frac{1}{2} \operatorname{Cov}\left(A_{m}, M\right)+\frac{1}{2} \operatorname{Cov}\left(A_{p}, M\right)
\end{aligned}
$$

qui, sachant que $\operatorname{Esp}\left(A_{p} / P\right)=h(h+$ es $) P$ et $\operatorname{Esp}\left(A_{m} / M\right)=h(h+$ es $) M$ donnent :

$$
\begin{aligned}
& \operatorname{Cov}\left(A_{d}, P\right)=\frac{1}{2} h(h+e s)\left[V_{p}+\operatorname{Cov}(P, M)\right] \\
& \operatorname{Cov}\left(A_{d}, M\right)=\frac{1}{2} h(h+e s)\left[V_{m}+\operatorname{Cov}(P, M)\right]
\end{aligned}
$$

si $A_{p}$ et $A_{m}$ étant fixés $P$ et $A_{d}$ (respectivement $M$ et $A_{d}$ ) deviennent indépendantes, en d'autres termes si $A_{p}$ et $A_{m}$ déterminent toute la liaison entre $P$ et $A_{d}$ (respectivement $M$ et $A_{d}$ ).

Par ailleurs :

$$
\operatorname{Esp}\left(\mathbf{R}_{\mathrm{d}} / \mathbf{A}_{\mathrm{d}}\right)=\frac{\operatorname{Cov}\left(\mathbf{A}_{\mathrm{d}}, \mathbf{R}_{\mathrm{d}}\right)}{\operatorname{Var}\left(\mathbf{A}_{\mathrm{d}}\right)} \mathbf{A}_{\mathrm{d}}=\frac{\mathrm{es}}{\mathrm{h}} \mathbf{A}_{\mathrm{d}}
$$

si $A_{d}$ étant fixe nous pouvons supposer que $P$ et $R_{d}$ (respectivement $M$ et $R_{d}$ ) sont indépendants,

d'où :

$$
\begin{aligned}
& \operatorname{Cov}\left(R_{d}, P\right)=\frac{e s}{h} \operatorname{Cov}\left(A_{d}, P\right) \\
& \operatorname{Cov}\left(R_{d}, M\right)=\frac{e s}{h} \operatorname{Cov}\left(A_{d}, M\right)
\end{aligned}
$$

$$
\begin{aligned}
& \operatorname{Cov}(\mathrm{P}, \mathrm{D})=\left(1+\frac{\mathrm{es}}{\mathrm{h}}\right) \quad \operatorname{Cov}\left(\mathrm{A}_{\mathrm{d}}, \mathrm{P}\right) \\
& \operatorname{Cov}(\mathrm{M}, \mathrm{D})=\left(1+\frac{\mathrm{es}}{\mathrm{h}}\right) \quad \operatorname{Cov}\left(\mathrm{A}_{\mathrm{d}}, \mathrm{M}\right)
\end{aligned}
$$

qui conduisent à :

$$
(\mathrm{h}+\mathrm{es})^{2}=\frac{2 \operatorname{Cov}(\mathrm{P}, \mathrm{D})}{\mathrm{V}_{\mathrm{p}}+\operatorname{Cov}(\mathrm{P}, \mathrm{M})}=\frac{2 \operatorname{Cov}(\mathrm{M}, \mathrm{D})}{\mathrm{V}_{\mathrm{m}}+\operatorname{Cov}(\mathrm{P}, \mathrm{M})}
$$

En présence d'une corrélation génotype-milieu l'estimation de $h^{2}$ proposée précédemment est donc surestimée de 2 hes $+\mathrm{e}^{2} \mathrm{~s}^{2}$. 
De la même façon entre diescendants d'un même parent, si l'on peut supposer labsence d'effets de milieu commun, les covarianoes entre $\mathbf{A}_{d}$ et $\mathbf{R}_{d}$, d'une part et entre $R_{d}$ et $R_{d^{\prime}}$ d'autre part seront alors dues exclusivement à la corrélation génotypemilieu.

On pourra écrire sachant que $\operatorname{Esp}\left(R_{d} / A_{d}\right)=\frac{e s}{h} A_{d}:$

$$
\begin{aligned}
\operatorname{Cov}\left(\mathbf{A}_{\mathrm{d}}, \mathbf{R}_{\mathrm{d}^{\prime}}\right) & =\frac{\text { es }}{\mathrm{h}} \operatorname{Cov}\left(\mathrm{A}_{\mathrm{d}}, \mathrm{A}_{\mathrm{d}^{\prime}}\right) \\
\text { et } \operatorname{Cov}\left(\mathbf{R}_{\mathrm{d}}, \mathbf{R}_{\mathrm{d}^{\prime}}\right) & =\left(\frac{\text { es }}{\mathrm{h}}\right)^{2} \operatorname{Cov}\left(\mathbf{A}_{\mathrm{d}}, \mathbf{A}_{\mathrm{d}^{\prime}}\right)
\end{aligned}
$$

dans ce cas :$$
\operatorname{Cov}\left(D, D^{\prime}\right)=\left(1+2 \frac{\mathrm{es}}{\mathrm{h}}+\frac{\mathrm{e}^{2} \mathrm{~s}^{2}}{\mathrm{~h}^{2}}\right) \operatorname{Cov}\left(\mathrm{A}_{\mathrm{l}}, \mathrm{A}_{\mathrm{d}^{\prime}}\right)
$$

et pour des descendants du même père :

$$
(\mathrm{h}+\mathrm{es})^{4}\left(2 \mathrm{~b}_{\mathrm{p} / \mathrm{m}}\right)+(\mathrm{h}+\mathrm{es})^{2}\left(1+\frac{\mathrm{V}_{\mathrm{p}}}{\mathrm{V}_{\mathrm{m}}}\right)-\frac{\mathrm{V}_{\mathrm{d}}}{\mathrm{V}_{\mathrm{m}}} \mathrm{V}_{\mathrm{d}}=0
$$

et pour des frères utérins :

$$
(\mathrm{h}+\mathrm{es})^{4}\left(2 \mathrm{~b}_{\mathrm{p} / \mathrm{m}}+\frac{\mathrm{V}_{\mathrm{p}}}{\mathrm{V}_{\mathrm{m}}} \mathrm{u}_{\mathrm{p}}\right)+(\mathrm{h}+\mathrm{es})^{2}-4 \frac{\mathrm{V}_{\mathrm{d}}}{\mathrm{V}_{\mathrm{m}}} \mathrm{u}_{\mathrm{l}}=0
$$

toutes trois équations du second degré en $(\mathrm{h}+\mathrm{es})^{2}$.

Par les méthodes d'analyse de la variance l'application des méthodes proposées précédiemment conduit donc également à surestimer l'héritabilité de 2 hes $+\mathrm{e}^{2} \mathrm{~s}^{2}$ en présence d'une corrélation génotype-milieu.

\section{Discussion et conclusion}

Le modèle génétique additif classique apparaît à la suite de cet exposé comme conduisant à suffisamment de complexité lorsque l'on s'éloigne de ses conditions habituelles d'application pour qu'en première approximation on se refuse à en utiliser de plus compliqué.

Répartissant la variance phénotypique observée en deux fractions indépendantes, l'une transmissible génétiquement et l'autre non transmissible, par le moyen de l'analyse de la ressemblance phénotypique entre individus apparentés, il permet dans les conditions de la panmixie de décrire la situation simplement à l'aide d'un seul paramètre, l'héritabilité. Ce sont ces conditions qui confèrent au modèle génétique additif classique sa plus grande efficacité. 
En élevage cependant, de plus en plus souvent, l'augmentation des informations disponibles sur les animaux conduit les éleveurs à gérer leurs populations dans des conditions parfois très éloignées de la situation panmictique de référence. D'autres paramètres doivent alors être introduits pour décrire ces nouvelles situations.

La prise en considération de la sélection amène à distinguer les variances phénotypiques des pères, mères et descendants $\left(\mathrm{V}_{\mathrm{p}}, \mathrm{V}_{\mathrm{m}}, \mathrm{V}_{\mathrm{d}}\right)$ au lieu de n'avoir affaire qu'à la seule variance phénotypique de la population.

La prise en considération de la structure des accouplements lorsqu'ils n'ont pas lieu au hasard, conduit à introduire la corrélation phénotypique entre conjoints (r) et même parfois la corrélation intra-classe de père du phénotype des conjoints $\left(\mathrm{t}_{\mathrm{m}}\right)$ ou des conjoints $\left(u_{p}\right)$ qui diffèrent parfois de $r^{2}$. On peut noter aussi dans ce cas que le modèle génétique additif est à manier avec précaution. On sait en effet (FisHer, 1918) qu'à l'équilibre des croisements assortis, la variabilité additive trouvée peut être sensiblement plus élevée que celle qui serait réalisée avec le même potentiel génétique dans des conditions panmictiques.

La prise en considération d'une répartition non aléatoire des effets de milieu conduit au minimum à introduire des effets de milieu commun généralement désignés par la notation $\mathrm{c}^{2}$. Lorsque de plus on a des raisons de supposer que ces effets sont en corrélation avec la valeur génétique additive, le moyen le plus simple de décrire la situation est d'introduire une corrélation génotype-milieu s qui permet avec un seul paramètre de grossièrement traduire une situation qui peut en fait être très complexe. En particulier si on a des raisons de supposer que les effets de milieu sur un individu dépendent en partie du phénotype d'un de ses parents, l'hypothèse d'une corrélation génotype-milieu ne peut être délibérément écartée. En outre, dans une situation de croisements raisonnés, étant donné (figures 1 et 2) l'ensemble des liaisons introduites, la probabilité de devoir en prendre une en considération est énormément accrue. Ceci nous amène tout naturellement à discuter de l'utilité de la formulation d'un tel modèle.

En tout premier lieu, je crois qu'une telle présentation des problèmes permet de mettre l'accent sur l'ensemble des hypothèses génétiques faites. Cela n'apparaît pas toujours de façon aussi évidente dans des formulations plus statistiques. C'est ainsi qu'elle permet d'élucider certaines difficultés concernant l'estimation de l'héritabilité dans des situations de croisements raisonnés et notamment dans le cas de la méthode de décomposition de la variance (LANGLoIs, 1975). De la même façon, le problème de l'évaluation des reproducteurs dans des situations non panmictiques mériterait d'être abordé sous ces deux angles complémentaires. Il importerait en particulier de ne pas négliger l'interprétation à faire en terme de modèle génétique des paramètres estimés dans le modèle statistique linéaire actuellement de plus en plus sophistiqué (HENDERSON, 1973).

Enfin l'introduction d'une corrélation génotype-milieu permet d'apprécier son incidence et amène à nuancer certains résultats. Mais il est souvent difficile d'apprécier son ordre de grandeur. Toutefois, dans les cas fréquents où l'on peut supposer que cette corrélation a pour origine un ajustement des effets de milieu sur les descendants en fonction des phénotypes parentaux, on peut envisager la mise en place de dispositifs expérimentaux pour l'estimer. Il faut dans ce cas pouvoir mesurer directement $\operatorname{Cov}\left(P, R_{d}\right)$ et $\operatorname{Cov}\left(M, R_{d}\right)$. Cela est envisageable dans les cas d'adoptions, d'erreurs ou de fraudes de pedigree ou bien de produits issus de transplantations d'ovules... 
Dans le cas de certains effets maternels où le phénotype de la mère détermine des effets de milieu sur les descendants, un certain nombre de questions que se pose le zootechnicien pourraient être résolues par l'application de ce modèle. En estimant par un dispositif expérimental l'importance et le signe de la corrélation génotype-milieu, on peut en effet répondre aux questions pratiques essentielles, à savoir : les effets maternels sont-ils liés de façon positive ou au contraire sont-ils antagonistes du caractère recherché et dans quelle mesure interfèrent-ils avec la sélection ? La confrontation des approches méthodologiques proposées dans ces domaines (FoulleY, LEFORT, 1978) avec la démarche proposée ici devrait conduire à d'utiles prolongements.

Reçu pour publication en mai 1981.

\section{Remerciements}

Nous remercions Messieurs Claude Chevalet et Jean-Louis Foulley, chercheurs du Département de génétique animale de l'I.N.R.A. de la lecture approfondie de ce texte et des critiques constructives qu'ils ont su formuler.

\section{Summary}

\section{Proposals for the extension of the conventional additive genetic model} to non panmictic situations

The results of FisHER (1918) on the correlation between relatives were extended to a number of situations which were often not taken into account such as non random matings in a selected population and genotype-environment correlation.

In a first step, taking into account only the additive genetic variability, the formulae derived from the work of FISHER for estimating heritability were confirmed and extended to a larger range of situations.

In a second step the introduction of a genotype-environment correlation allowed to estimate its effect on the former estimations.

It was concluded on the usefulness of such a model which leads to clarify the genetic hypothesis and to a better approach of the problems of estimation of breeding values. In cases where a genotype-environment correlation cannot deliberately be discarded it allows sometimes to estimate it and otherwise to evaluate its possible effect on the results. Moreover this approach leads to examine in an original way the analysis of some maternal effects.

\section{Références bibliographiques}

Fisher R.A., 1918. The correlation between relatives on the supposition of Mendelian inheritance. Trans. r. Soc. Edinb., 52, 399-433.

Foulley J.-L., Lefort G., 1978. Revue bibliographique. Méthodes d'estimation des effets directs et maternels en sélection animale. Ann. Génét. Sél. anim., 10, 475-496. 
Henderson C.R., 1973. Sire evaluation and genetic trends. In: Proc. Anim. Breed. Genet. Symp., Blacksburg, Virgina, pp. 10-41. American Society of Animal Science, Champaign, Illinois.

JENCKS C., 1972. In: Inequality (Ed. Harper et Row, New York et Londres). Appendix A : Estimating the heritability of IQ scores, 266-319.

Langlois B., 1975. Analyse statistique et génétique des gains des Pur-Sang anglais de trois ans dans les courses plates françaises. Ann. Génét. Sél. anim., 7, 387-408.

Langlors B., 1980. Heritability of racing ability in Thoroughbreds. A review. Livest. Prod. Sci., 7, 591-605.

REEvE E.C.R., 1953. Studies of quantitative inheritance III. Heritability and genetic correlation in progeny tests using different mating systems. J. Genet., 51, 520-542.

Refve E.C.R., 1955. Contribution to discussion. Cold. Spr. Harb. Symp. Quant. Biol., 20, 76-78. 